\title{
Breve revisão sobre uso de fauna medicinal no Brasil: aspectos históricos, farmacológicos e conservacionistas
}

\author{
Wedson Medeiros Silva Souto ${ }^{1 *}$, Washington L. S. Vieira ${ }^{1}$, Paulo Fernando Guedes Montenegro ${ }^{2}$, \\ Humberto Nóbrega Alves ${ }^{3}$, Rômulo Romeu Nóbrega Alves ${ }^{4}$
}

${ }^{1}$ Programa de Pós-graduação em Ciências Biológicas (Zoologia) e 2 Laboratório de Ecofisiologia Animal, Departamento de Sistemática e Ecologia, Universidade Federal da Paraíba, 58059-970, João Pessoa, Paraíba, Brasil.

${ }^{3}$ Prefeitura Municipal de João Pessoa, Escola Municipal Arnaldo de Barros Moreira, Rua Capitão Francisco Pereira, 365, Bairro dos Novais, 58088-530, João Pessoa, Paraíba, Brasil.

${ }^{4}$ Departamento de Biologia, Universidade Estadual da Paraíba, Avenida das Baraúnas, Campina Grande, 58109-753, Paraíba, Brasil.

\begin{abstract}
Resumo - O tratamento de doenças em humanos e em animais por meio do uso de remédios obtidos de animais ou de partes destes é conhecido como zooterapia. Apesar de constituir uma prática relativamente comum, estudos sobre este tema são relativamente escassos quando comparados àqueles que abordam a temática de remédios tradicionais obtidos de plantas. No Brasil, já foram registrados 326 animais explorados para finalidades medicinais, a maioria das quais é silvestre. A literatura acerca dos usos de animais medicinais é numericamente bastante inferior aos registros da utilização de plantas medicinais e suas implicações, o que potencializa a necessidade do desenvolvimento de mais pesquisas sobre o tema. Entre os diversos fatores que ressaltam a importância de uma maior compreensão da zooterapia, destacam-se: perspectivas históricas, aspectos relacionados à conservação e à validação desses medicamentos tradicionais e dificuldades no desenvolvimento de pesquisas sobre fauna medicinal. No presente trabalho, nós construímos uma breve discussão sobre cada um destes tópicos.
\end{abstract}

Palavras-chave adicionais: conservação, etnozoologia, zooterapia.

\begin{abstract}
A brief review of the medical use of fauna in Brazil: conservationist, historical, and pharmacological aspects) The treatment of human and animal aliments by using drugs based on extracts obtained from animals, or ultimately derived from them, is known as zootherapy. Although this is a relatively common practice, studies on this subject are relatively scarce when compared to those that are focused on traditional plant-based medicines. In Brazil, 326 animals are exploited for medicinal purposes, most of which are wild species. The number of literature records on the medicinal uses of animals is much lower than those on the use of medicinal plants and their implications, which suggests the need to develop more research on the topic. Among the many factors that highlight the importance of a greater understanding of zootherapy, are: historical perspectives, aspects related to conservation, the validation of traditional medicines, and the difficulties in developing research on the medicinal fauna. In this study, we provide a brief discussion of each topic.
\end{abstract}

Additional key words: conservation, ethnozoology, zootherapy.

Os animais constituem uma parte de nossa cultura e são utilizados de várias maneiras para satisfazer as necessidades humanas (van den Bos \& de Jonge 2005). A diversidade de interações que as culturas humanas mantêm com os animais tanto pode ser estudada do ponto de vista das disciplinas da ciência ocidental, tais como zoologia, psicologia e etologia, quanto pela perspectiva da etnociência, mais particularmente da etnozoologia (CostaNeto 2000; Alves et al. 2006; Alves \& Souto 2010a). Embora pinturas rupestres e determinados registros arqueológicos possam ser considerados em essência dados etnozoológicos (ver Baker 1941), documentos escritos, no entanto, é que possibilitaram registrar mais precisamente as informações sobre as interações de grupos humanos antigos e os usos que eram feitos da fauna (Alves \& Souto 2010a).

\footnotetext{
*Autor para correspondência: wedson@live.com Editora responsável: Maria Franco Trindade Medeiros

Recebido 16 maio 2011; aceito 5 jul. 2011.
}

Uma das formas de interação entre humanos e a fauna registrada nos documentos históricos se refere ao uso medicinal de animais ou seus derivados para tratamento de doenças (Lev 2000, 2003). Esse tipo de utilização da fauna se perpetua até os dias atuais, constituindo uma prática hoje conhecida como zooterapia (Marques 1997; Alves \& Rosa 2005; Costa-Neto 2005a), um fenômeno que é marcado tanto por uma ampla distribuição geográfica quanto por origens históricas profundas (Alves 2008a; Costa-Neto \& Alves 2010a), o que levou Marques (1994) a publicar a hipótese da universalidade zooterápica, segundo a qual toda sociedade humana que apresenta um sistema médico utiliza remédios feitos à base de animais.

Inserido neste contexto, a descoberta das Américas pelos Europeus abriu um vasto mundo novo de vida vegetal e animal. Os trabalhos de observadores antigos, apesar de terem considerados os povos nativos como desprovidos de conhecimento, incluíram muita informação importante acerca dos usos indígenas do ambiente, especialmente 
plantas, e desta forma constituem base para estudos etnohistóricos. De fato, a adaptação de vários grupos humanos aos mais diversos recursos biológicos gerou incalculáveis sistemas de conhecimento que incluem uma extensiva informação sobre os usos da fauna em geral e, em particular, das espécies medicinalmente úteis (Alves \& Rosa 2007a). Ao longo dos séculos, animais ou partes destes têm sido amplamente usados na medicina tradicional brasileira e têm desempenhado um significativo papel em práticas de tratamento e cura de enfermidades (Almeida 2005; Alves \& Rosa 2006; Alves et al. 2007).

Publicações recentes demonstraram a importância da zooterapia em vários ambientes socioculturais. Inúmeros exemplos do uso de remédios derivados de animais podem ser encontrados em muitas áreas urbanas, semiurbanas e localidades remotas em todas as partes do mundo, especialmente em países em desenvolvimento (Mahawar \& Jaroli 2006, 2007; Vázquez et al. 2006; El-Hani \& Bandeira 2008; Alves 2009; Alves et al. 2009a,b; Ferreira et al. 2009a; Quave et al. 2010), onde a medicina tradicional é praticada por $80 \%$ da população (World Health Organization 2002).

Apesar da prevalência das práticas médicas tradicionais em um contexto histórico e contemporâneo, pesquisas acerca de animais medicinais têm sido frequentemente negligenciadas em comparação com o uso de plantas medicinais (Alves et al. 2009a; Quave et al. 2010). Isto pode ser facilmente retratado numericamente quando analisamos a produção científica sobre o uso de recursos biológicos medicinais. Enquanto Calixto (2005) registrou 3.722 trabalhos publicados acerca de plantas medicinais, Alves \& Souto (2010b) encontraram 72 publicações em zooterapia no Brasil. De uma perspectiva farmacológica, a situação não é diferente. Pieroni et al. (2002), por exemplo, destacam que estudos sobre drogas de origem animal são ainda raros na literatura científica. Nesse contexto, estudos sobre o uso tradicional de animais medicinais podem fornecer subsídios para a realização de ensaios farmacológicos (Alves \& Rosa 2005).

Entre os diversos fatores que ressaltam a importância de uma maior compreensão da zooterapia, destacam-se: perspectivas históricas, aspectos relacionados à conservação e à validação desses medicamentos tradicionais e dificuldades no desenvolvimento de pesquisas sobre fauna medicinal. No presente trabalho, nós endereçamos uma breve discussão sobre cada um destes tópicos.

\section{Perspectivas Históricas}

Em regiões neotropicais, muito da informação acerca dos modos de vida tradicionais de caçadores e coletores anteriores à transformação ou destruição de suas terras, subsequente a invasão europeia - veio de escritos de cronistas, missionários, comerciantes e exploradores (Ingold
1994). Documentos históricos relevantes trazem informações acerca do conhecimento dos habitantes do Brasil colonial. Por exemplo, Gabriel Soares de Sousa, em Tratado Descriptivo do Brazil em 1587, já indicava áreas viáveis à ocupação, baseando-se na disponibilidade de recursos, incluindo animais de caça (Sousa 1851). Embora não estivessem detalhadas quais as espécies preferenciais de caça, isto indica que os portugueses já haviam assimilado com os indígenas quais daquelas seriam úteis para diversos fins, inclusive medicinal (ver Sousa 1851). Naturalistas nassovianos do século XVII, tais como Georg Marcgrave e Guilherme Piso (Willem Piso, em holandês), além de produzirem obras históricas acerca da composição faunística e florística do Novo Mundo, contribuíram indiretamente para o registro de informações etnozoológicas importantes. Nas obras seiscentistas de Piso, História Natural do Brasil Ilustrada e História Natural e Médica da Índia Ocidental, somente traduzidas para o português no final da década de 1940 e início da década de 1950, há relatos de 29 animais medicinais (Almeida 2005), espécies tanto nativas quanto exóticas (e.g., o preá Coendou prehensilis (Linnaeus, 1758) e o boi Bos taurus Linnaeus, 1758, respectivamente), indicando que neste tempo já ocorrera permuta de conhecimento etnozoológico entre nativos e europeus.

Almeida (2010) destaca que há importantes obras entre os séculos XVI e XIX que mencionam usos de animais medicinais, mesmo em obras de ilustres figuras da medicina, entre os quais se destacam Moquin-Tandon (1861) e Stephenson (1832) que empregaram o termo "medical zoology" (zoologia médica). Ainda de acordo com Almeida (2010), no Brasil do século XVII, os médicos portugueses Simão Pinheiro Morão (c. 1618-1685) e João Ferreyra da Rosa (c. 1659-1725) produziram tratados médicos com receituários de 22 e 19 espécies zooterapêuticas, respectivamente. No entanto, muitas espécies da fauna empregadas medicinalmente em tempos coloniais permanecem desconhecidas, pois, como destaca Alencastro (2000), os médicos seiscentistas portugueses assimilaram as doenças tropicais americanas à nosologia acadêmica europeia, mas rejeitavam os medicamentos indígenas e as práticas de cura afro-brasileiras.

$\mathrm{Na}$ América Latina, as culturas indígenas vivem junto aos demais povos americanos, de tal forma que descendentes de africanos, italianos, alemães, poloneses e outros imigrantes, os quais vieram posteriormente ou foram trazidos pelos colonizadores originais portugueses ou espanhóis para trabalharem em terras (Fragoso et al. 2004), originaram uma diversidade cultural ímpar. Durante os mais de 500 anos de mistura, membros destes grupos criaram uma etnicidade latino-americana vital, com cada país exibindo uma descendência única que, apesar das ligações com as visões de mundo europeia e cristã, é também profundamente enraizada nas condições ambientais locais e nas paisagens (Pratt 1992). De certo, os países latinoamericanos possuem uma diversidade cultural ímpar, o que 
estimula a realização de pesquisas antropológicas e etnobiológicas, tanto em estudos de campo quanto pesquisas que visem a uma documentação de registros bibliográficos históricos acerca da fauna medicinal.

\section{Zooterapia e Conservação}

Os medicamentos tradicionais permanecem como um legado valioso do passado, especialmente nos países em desenvolvimento, onde reside a maior parte da população mundial, bem como em países industrializados, onde os medicamentos tradicionais rendem bilhões de dólares por ano (Farnsworth 1988; Fabricant \& Farnsworth 2001). No entanto, os impactos das práticas zooterapêuticas sobre as populações silvestres necessitam ser cuidadosamente avaliados, particularmente considerando que, diferente de muitos remédios derivados de plantas, a preparação da maioria dos remédios derivados de animais são produtos obtidos após a morte do espécime (Alves \& Rosa 2006; Ashwell \& Walston 2008). Neste sentido, Almeida \& Albuquerque (2002) apontam para a necessidade de conscientização sobre o uso sustentável de animais medicinais.

Sabe-se que existe um predomínio de animais silvestres como fontes de remédios tradicionais (Alves et al. 2007, 2008; Van \& Tap 2008; Souto et al. 2011). No Brasil, dados de 2007 indicaram que $96 \%$ dos animais explorados medicinalmente são silvestres (ver Alves et al. 2007) e este valor praticamente permanece inalterado, apesar do registro do uso de animais medicinais ter aumentado de 283 espécies para 326 espécies (Costa-Neto \& Alves 2010a). Portanto, a tendência do uso predominante de espécies da fauna silvestre na medicina popular brasileira é uma realidade. Embora o número de animais inventariado seja expressivo; o mesmo deve ser ainda maior, uma vez que as pesquisas sobre o tema ainda são incipientes, evidenciando a necessidade de estudos adicionais buscando inventariar a fauna medicinal de maneira mais completa (Alves et al. 2007; Alves 2008b).

Adicionalmente, o uso de animais para propósitos medicinais é causa de preocupação para algumas espécies (Lee et al. 1998). O uso insustentável de plantas e animais na medicina tradicional é reconhecido como uma ameaça à conservação da vida selvagem (Alves et al. 2009c). A ligação entre as espécies ameaçadas e a demanda da medicina tradicional tem despertado muita preocupação da comunidade científica mundial (Brautigam et al. 1994; Reed 1995; Alves \& Rosa 2007a). O aumento do uso de animais medicinais tem conduzido, por exemplo, à sobre-exploração de espécies como rinocerontes, tigres, cervos, ursos, macacos e pangolins, sobretudo na Ásia e na África (Miller 1993; Hunter Jr \& Gibbs 2007). No Camboja, o tigre [Panthera tigris (Linnaeus, 1758)] e o pangolim-de-Sunda (Manis javanica Desmarest, 1822) tornaram-se ameaçados principalmente devido à cultura local do uso dessas espécies na medicina tradicional (ver Ashwell \& Walston 2008). Conservacionistas têm aumentado a preocupação pelo comércio de produtos animais utilizados como ingredientes para diversos remédios na medicina tradicional asiática. Por exemplo, ossos de tigre são usados para tratar reumatismo, fraqueza e paralisia (Mills \& Jackson 1994) e, embora o uso e comércio de partes de tigre tenham sido banidos há mais de uma década, o comércio ilegal ainda continua (Loveridge et al. 2010). Partes de outros felídeos (leopardos, leões, entre outros) estão sendo utilizados como substitutos para remédios obtidos de partes de tigres e, portanto, também constituem impactos sobre a população dessas espécies (Wingard \& Zahler 2006). Ainda na Ásia, muitas espécies de langures (Primatas) estão agora severamente ameaçadas, não apenas em virtude da caça para subsistência e perda de hábitat, mas também em virtude da caça para uso de partes e tecidos a fim de satisfazer a demanda por pratos exóticos e preparos de animais silvestres para amuletos, remédios e afrodisíacos (Mittermeier et al. 2007).

ALista Vermelha de Espécies Ameaçadas (IUCN 2010) é considerada o índice de maior status de conservação das espécies, em especial de vertebrados (Schipper et al. 2008) e sofre grandes atualizações frequentemente; a última foi em 2010. As categorias da lista vermelha são atribuídas com base em critérios quantitativos, tais como limites de tamanho da população, a taxa de declínio ou a probabilidade de extinção (Macdonald et al. 2010). Estudos recentes demonstram que os impactos da medicina tradicional atingem diversos grupos de animais (Costa-Neto 2005b; Costa-Neto \& Alves 2010b; Alves \& Alves 2011). Contudo, os vertebrados normalmente são aqueles mais explorados, uma vez que constituem fontes de várias partes distintas empregadas como remédios (Alves \& Rosa 2006, 2007b; Mahawar \& Jaroli 2007, 2008; Quave et al. 2010; Souto et al. 2011). Alves et al. (2010a) elaboraram uma revisão global do uso de primatas da medicina tradicional e perceberam que todas as espécies exploradas medicinalmente estão listadas na lista vermelha. Em relação aos canídeos, 55\% ( $\mathrm{n}=19)$ das espécies descritas atualmente são exploradas para propósitos medicinais em várias partes do mundo e também estão na lista da IUCN (Alves et al. 2010b).

Neste contexto, a importância de registros dos usos tradicionais de recursos faunísticos tem sido considerada por organismos internacionais, a exemplo da TRAFFIC (ver Shepherd 2001; Debbie \& Burgess 2005; Walston 2005) e da própria IUCN. De acordo com a IUCN, 14\% das aves ameaçadas do mundo são exploradas para obtenção de alimentos ou remédios e este número está provavelmente subestimado (Vié et al. 2009). Embora 12\% de todas as espécies de aves estejam globalmente ameaçadas de extinção, uma larga proporção $(23 \%)$ das espécies usadas como fontes de alimentos e remédios está ameaçada (Vié et al. 2009). No caso dos répteis, a sobre-exploração é um problema que não afeta tão somente tartarugas, mas também 
várias espécies de lagartos e cobras, cujo declínio de suas populações tem sido verificado de forma intensa na América tropical (Fitch et al. 1982). Para este grupo, sabe-se que pelo menos 165 espécies de répteis são usadas na medicina tradicional em várias partes do mundo, embora a medicina tradicional não constitua necessariamente a principal forma de exploração e impacto sobre estes animais (Alves et al. 2008).

De fato, a medicina tradicional deve ser compreendida como um dos vários fatores que levam determinadas espécies a se tornarem ameaçadas. Muitas das espécies silvestres exploradas para alimento e remédios estão agora ameaçadas de extinção, algumas devido à sobre-exploração, outras em virtude de diferentes pressões, tais como a perda de hábitat, doenças ou uma combinação de fatores (TRAFFIC 2010). A perda de hábitat decorrente da expansão das áreas agrícolas e desmatamento têm sido os principais responsáveis por uma maior perda de biodiversidade (Richmond-Coggan 2006; Wade 2008; Allebone-Webb 2009; IUCN 2009, 2010). No entanto, para alguns grupos, os impactos decorrentes da exploração para fins medicinais são algumas vezes equiparáveis à perda de hábitat. Por exemplo, o uso medicinal não é considerado a maior preocupação para a conservação dos canídeos e nenhuma das espécies deste grupo listadas como ameaçadas pelo IUCN tiveram seu uso destacado como a principal ameaça para suas populações (Alves et al. 2010b). O mesmo não ocorre para algumas espécies de felinos e outras espécies ameaçadas, as quais têm sido historicamente usadas em formulações tradicionais, a exemplo de primatas, rinocerontes, ursos e cavalos marinhos (Mills \& Jackson 1994; Ellis 2005; Dollo et al. 2010; IUCN 2010; Nijman 2010). $\mathrm{O}$ uso medicinal da fauna cria, deste modo, uma ameaça adicional e deve ser considerada em conjunto com outras pressões antropogênicas (Alves et al. 2010b). As espécies medicinais cujo status de conservação estão em questão devem receber urgente atenção e considerações relacionadas a todos os fatores que contribuem para extirpações e suas conexões com os usos medicinais atuais e futuros (Alves et al. 2007; Oliveira et al. 2010).

Embora na América Latina o uso medicinal não seja tão impactante quanto na Ásia, exemplos de espécies silvestres cujas populações sofrem uma pressão adicional em virtude da exploração para fins medicinais têm sido registrados nos últimos anos. Lamentavelmente, alguns recursos zooterapêuticos usados no Brasil, por exemplo, estão incluídos em listas oficiais de espécies ameaçadas (IBAMA 2003; IUCN 2010). Alves (2009), por exemplo, registrou que $92 \%(n=230)$ dos animais usados na etnomedicina do Nordeste do Brasil $(\mathrm{n}=250)$ são silvestres e inclui espécies vulneráveis, tais como o cavalos-marinho Hippocampus erectus Perry, 1810, a tartaruga-cabeçuda Caretta caretta (Linnaeus, 1758), a tartaruga-verde Chelonia mydas (Linnaeus, 1758), o zabelê Crypturellus noctivagus (Wied, 1820), o tatu-bola Tolypeutes tricinctus
(Linnaeus, 1758), além de outras criticamente ameaçadas, tais como o peixe-boi Trichechus manatus (Linnaeus, 1758) e o macaco guariba Alouatta belzebul (Linnaeus, 1766). Até 2007, pelo menos 75 espécies da fauna empregadas na farmacopeia tradicional brasileira estavam listadas em alguma lista de espécies ameaçadas (Lista Vermelha da IUCN, Lista Brasileira de Espécies Ameaçadas e Lista Nacional das espécies de invertebrados aquáticos e peixes ameaçadas, sobre-explotadas ou ameaçadas de sobreexplotação) (Alves et al. 2007), muito embora as razões para estas inclusões não necessariamente estão relacionadas ao uso medicinal.

A depleção de recursos medicinais não apenas impõe um desafio para a conservação, mas representam uma séria ameaça para a saúde de muitas comunidades humanas. Esforços para estabilizar o status dessas espécies são importantes não apenas para os conservacionistas, mas para milhões de pessoas cujo tratamento de doenças e enfermidades depende do uso de remédios tradicionais (Still 2003; Alves et al. 2010a,b). Moura \& Marques (2008) destacaram que uma das características do uso da fauna medicinal é que os produtos zooterapêuticos de animais inteiros ou suas partes não servem para outros propósitos. Neste sentido, é notável que na maioria dos casos os produtos medicinais de animais são subprodutos de animais caçados para outros propósitos; assim, estes múltiplos usos (incluindo medicinal), da fauna e seus impactos nas populações devem ser devidamente avaliados e tomados em consideração quando planos de recuperação para estas espécies forem implementados, especialmente para aquelas que são frequentemente exploradas (Alves \& Rosa 2006, 2007a,b; Alves et al. 2007, 2010a)

Estratégias devem ser pensadas para conciliar tanto as práticas zooterapêuticas quanto o manejo de espécies exploradas para propósitos medicinais. É importante que as futuras pesquisas sobre zooterapia busquem identificar se os remédios derivados de animais (sobretudo de espécies ameaçadas) possuem substitutos obtidos de plantas ou de animais domésticos também utilizados na farmacopeia tradicional. Alguns autores (Alves \& Rosa 2006; Alves et al. 2007) já indicaram que há sobreposição entre usos de plantas e animais medicinais na etnomedicina brasileira. Este aspecto abre uma perspectiva de, quando sustentável, substituir o uso de espécies ameaçadas por outras em receituários médicos tradicionais. Paralelo a isto, no entanto, torna-se necessário que sejam conduzidas pesquisas de validação tanto do remédio original quanto de seu substituto, já que, segundo Sodeinde \& Soewu (1999), a substituição de ingredientes em remédios deve ser conduzida com cuidado porque os substitutos podem nem sempre ter a mesma eficácia.

Muitas espécies da fauna medicinal deparam-se sob risco de extinção, o que poderá acarretar uma perda detalhada de informação etnozoológica a curto prazo. Para a maioria daquelas ameaçadas não estão sendo tomadas 
ações que de fato visem a conservação. Igualmente, pouca ênfase tem sido colocada no potencial de descoberta de novas drogas, e muito pouco tem sido destacado sobre os problemas envolvidos no uso de medicamentos tradicionais das populações locais, a exemplo do risco de contaminação biológica e apropriação do conhecimento tradicional. Desta forma, é importante que pesquisas direcionadas para se a verificar a real eficácia dos remédios.

Além do mais, como apontado por Marques (1997), o potencial médico da fauna brasileira e a possibilidade de exaustão por meio da sobre-exploração ou uso de espécies em extinção requerem urgentemente uma avaliação ecológica deste processo não somente pela avaliação de sua dimensão zoológica, mas também por sua dimensão cultural. A compreensão de quais espécies são importantes do ponto de vista etnomedicinal em cada região do país emerge como uma forma de compreender quais animais são culturalmente importantes nas práticas tradicionais de medicina existentes. Esta observação é plenamente sustentada pelo fato de que estudos prévios já demonstraram que a diversidade de animais medicinais usados por populações humanas é influenciada pela composição faunística regional (Alves \& Rosa 2006, 2007a,b).

Programas educacionais podem ser usados para promover o uso de alternativas, focando comunidades onde o uso de animais silvestres para fins medicinais persiste. Em algumas circunstâncias, crenças locais, doutrinas religiosas e tabus específicos podem ser importantes para a conservação de espécies que estão ameaçadas ou em declínio, daí elas devem ser compreendidas e estimuladas (Alves et al. 2010a)

\section{Potencial Farmacológico}

Aceita-se amplamente que usos médicos tradicionais ou populares (informação etnomedicinais e/ou etnoveterinárias) de recursos indiquem a presença de um constituinte biologicamente ativo (Costa-Neto 1999; Tabuti et al. 2003; Rochfort et al. 2008; Alves et al. 2009a). Um grande número de produtos naturais é obtido a partir do estudo científico dos remédios tradicionalmente usados por diferentes culturas (Holmstedt \& Bruhn 1983). Quase um quarto de todos os medicamentos modernos é derivado de produtos que foram usados inicialmente como remédios tradicionais (Shetty 2010). Drogas importantes, tais como a artemisinina, o ascaridol, a podofilotoxina e a vincristina, foram descobertas a partir da investigação de usos médicos tradicionais (Liu et al. 2007; Patwardhan 2009).

Animais têm sido metodologicamente testados por companhias farmacêuticas como fontes de drogas para a medicina moderna (Kunin \& Lawton 1996). Atualmente, a porcentagem de animais fontes para a produção de medicamentos essenciais é igualmente significativa (Alves \& Rosa 2005). O uso prolongado e contínuo de uma espécie particular em uma determinada cultura para tratar um tipo particular de doença pode fornecer uma demonstração de eficácia (Soejarto 1996). Em outras palavras, usos médicos tradicionais ou populares podem encurtar o caminho para a descoberta de novas drogas (Alves \& Rosa 2006, 2007b). Contudo, apesar de existir uma relevante base de dados acerca da fauna medicinal brasileira, consolidada especialmente nos últimos cinco anos (Alves et al. 2007), poucas pesquisas estão sendo direcionadas para validar o uso de remédios derivados de animais e, como atesta Souto et al. (2009), desconhece-se quais dos muitos animais da farmacopeia tradicional brasileira são realmente eficientes para o tratamento de doenças.

Recentemente, Ferreira et al. (2010) avaliaram a atividade anti-inflamatória do uso tópico de banha (gordura) de teiú [Tupinambis merianae (Duméril \& Bibron, 1839)] vem modelos animais (machos e fêmeas de ratos $M u s$ musculus Linnaeus, 1758). Nesta primeira evidência de atividade anti-inflamatória de banha deste réptil em modelos in vivo, os autores encontraram que a banha tem importante atividade anti-inflamatória e que reduz o edema nas orelhas de ratos causados por óleo de cróton, fenol e ácido araquidônico. Em outro estudo, Ferreira et al. (2011) descobriram uma atividade sinergística da banha de jibóia Boa constrictor Linnaeus, 1758, quando combinada com um antibiótico, embora o zooterapêutico sozinho não tivesse qualquer atividade bactericida.

Além da importância de identificação de propriedades medicinais válidas, novos estudos são também necessários para estabelecer se os animais medicinais possuem efeitos adversos. Análises de risco/benefício são necessárias para serem aplicadas aos medicamentos zooterapêuticos. Sabese que alguns produtos naturais (normalmente de plantas, animais e minerais) usados na medicina tradicional podem causar sérios efeitos adversos (De Smet 1991). Ingredientes frequentemente usados na preparação de remédios tradicionais, tais como banhas, ossos, peles e couros, bile, cabeça e olhos, além de resultarem na morte de animais, também preocupam acerca das condições ideais de armazenamento e esta questão deve ser tratada em matéria de Saúde Pública.

Numerosas doenças infecciosas podem ser transmitidas entre animais e de animais para humanos (i.e., zoonoses) (Alves \& Rosa 2005; Kahn 2006) e esse contexto deve ser seriamente considerado nos âmbitos das mais diversas formas de medicina tradicional (Still 2003). Diversos órgãos e tecidos, incluindo ossos e bile, podem ser fonte de infecções de Salmonella, causando diarreia e choque endotóxico (Alves et al. 2009d). A possibilidade de transmissão de outras zoonoses graves e variadas, a exemplo de tuberculoses e raiva, deve ser levada em conta sempre que os tecidos animais provenientes de fontes desconhecidas são tratados e utilizados como remédios (Schnurrenberger \& Hubbert 1981). Meningites causadas por bactérias do gênero Streptococcus ou infecções de 
Campylobacter e Streptobacillus também podem ser transmitidas de animais para humanos e outros animais (Kahn 2006).

\section{Desafios e Dificuldades}

Vários problemas interpõem dificuldades para a realização de pesquisas que tenham por objetivo documentar as práticas zooterapêuticas, compreender o universo sociocultural associado a esse fenômeno, bem como para testar os remédios derivados de produtos animais. Semelhante a toda pesquisa etnobiológica, qualquer pesquisador que pretende desenvolver estudos nessa área, uma vez que se trata de um campo de pesquisa multidisciplinar, necessita do domínio de conceitos e métodos das ciências naturais e humanas (Alves \& Souto 2010c). Além disso, as interfaces estabelecidas com áreas tais como Biologia da Conservação, Farmacologia e Antropologia poderão exigir a participação de vários pesquisadores e alunos na condução de um projeto amplo.

Embora a legislação brasileira proíba o uso e comercialização da fauna silvestre (Lei 5.197, Artigo $1^{\circ}$, de 3 de janeiro de 1967; Lei 9.605, Artigo 12, de 12 de fevereiro de 1998), produtos e derivados de animais são comumente usados e comercializados em mercados tradicionais, os quais representam importantes pontos de venda da fauna nativa exótica ou local em diferentes regiões do país (Alves \& Santana 2008; Alves et al. 2009e; Ferreira et al. 2009b; CostaNeto \& Alves 2010b). As dificuldades de coleta de dados são ampliadas à medida que as pessoas, com receio da atuação e fiscalização de órgãos ambientais estaduais ou federais, temem participar das pesquisas etnobiológicas por confudirem professores ou estudantes como agentes disfarçados. De acordo com Alves e Souto (2010c), a situação torna-se mais difícil quando a pesquisa se desenvolve em áreas urbanas nas quais o comércio de animais silvestres comumente ocorre em mercados públicos e feiras livres. Nessas localidades, as fiscalizações são mais frequentes, resultando em apreensão de produtos derivados de animais silvestres que são comercializados para diferentes finalidades, sendo noticiadas em jornais locais.

Além dos problemas citados, outro desafio está em assegurar um rigor e uma precisão taxonômica das espécies exploradas localmente. Nomes populares variam bastante dependendo da localidade, de forma que a correspondência de um nome comum com um nome científico não permite uma identificação confiável (ver Mead 1970). A ausência de material-testemunho é a principal causa da falta de rigor científico em muitos estudos etnobiológicos (Nguyen 2005). Assim, numa perspectiva atual e futura, tais pesquisas, bem como periódicos científicos onde são publicadas, apontam para a necessidade de coleta de espécimes-testemunho e depósito em coleção científica, sendo esse um dos passos fundamentais na busca da melhoria qualitativa dos estudos de etnozoologia. A identificação correta no nível de espécie fornece uma base para comparação de dados biológicos, ecológicos e etnozoológicos (Bye Jr 1986; Hunn 1992).

Sempre que possível, seja por meio de coletas ativas, usos de armadilha ou doações, pelo menos um exemplar ou amostra de partes corporais de cada espécie deve ser coletado. Após este procedimento, os espécimes devem ser adequadamente rotulados e conservados em coleções científicas.

\section{Conclusões}

A zooterapia constitui um campo relevante de pesquisas etnozoológicas. Embora os trabalhos sobre o tema tenham se intensificado recentemente, mais estudos serão necessários para suprir uma lacuna existente acerca das informações da fauna medicinal brasileira, tanto em uma perspectiva histórica quanto sobre os aspectos culturais, farmacológicos e ecológicos. É importante que estas pesquisas sejam conduzidas em perspectiva multidisciplinar para suprir o maior número possível de dados.

No Brasil, como em outros países, a maioria das espécies animais usadas para propósitos medicinais compreende animais silvestres, sendo usualmente necessária a morte dos animais para obtenção dos produtos zooterápicos. Embora algumas das espécies medicinais estejam ameaçadas e o uso medicinal seja um fator adicional de depressão sobre essas espécies, o impacto da zooterapia necessita ser mais bem avaliado, sobretudo quando se comparam tais usos a outros fatores, como a degradação de hábitat e captura de animais para outros fins que não medicinais, causas evidentes do declínio populacional de muitas espécies. Todavia, assim como outros temas que envolvem pesquisas com fauna silvestre e moradores locais, uma maior integração e participação de professores pesquisadores e alunos será necessária para adquirir um grau de confiança suficiente para a condução dos estudos.

Os aspectos socioculturais são relevantes quando discutimos desenvolvimento sustentável. Esta perspectiva social inclui o modo como pessoas se tornam conscientes dos recursos naturais, de sua utilização, alocação, transferência e manejo. Assim, a inclusão da zooterapia na multidimensionalidade do desenvolvimento sustentável é interpretada como um componente fundamental para alcançar o uso sustentável dos recursos faunísticos. Novos estudos sobre a fauna medicinal, que buscam uma melhor compreensão desta forma de terapia, incluindo aspectos ecológicos, culturais e farmacológicas, são necessários. 
Alencastro, L.F. 2000.O Trato dos Viventes: formação do Brasil no Atlântico Sul. Companhia das Letras, São Paulo.

Allebone-Webb, S.M. 2009. Evaluating Dependence on Wildlife Products in Rural Equatorial Guinea. Ph.D. Thesis. University of London.

Almeida, A.V. 2005. Prescrições zooterápicas indígenas brasileiras nas obras de Guilherme Piso (1611-1679). In: A.G.C. Alves, R.F.P. Lucena \& U.P. Albuquerque (eds.), Atualidades em Etnobiologia e Etnoecologia. Sociedade Brasileira de Etnobiologia e Etnoecologia, NUPEEA, Recife, p. 47-60.

Almeida, A.V. 2010. A zooterapia adotada pelos médicos Simão Pinheiro Morão (c. 1618-1685) e João Ferreyra da Rosa (c. 1659-1725) em Pernambuco no final do século XVII. In: E.M. Costa-Neto \& R.R.N. Alves (eds), Zooterapia: os animais na medicina popular brasileira. NUPEEA, Recife, p. 55-74.

Almeida, C.F.C.B.R. \& Albuquerque, U.P. 2002. Uso e conservação de plantas e animais medicinais no estado de Pernambuco (Nordeste do Brasil): um estudo de caso. Interciencia 27: 276-285.

Alves, M.S.; Silva, M.A.; Júnior, M.M.; Paranaguá, M.N. \& Pinto, S.L. 2006. Zooartesanato comercializado em Recife, Pernambuco, Brasil. Revista Brasileira de Zoociências 8: 99109.

Alves, R.R.N. 2008a. Animal-based remedies as complementary medicine in Brazil. Forsch Komplementmed 15: 226-227.

Alves, R.R.N. 2008b. Commercialization of Uranoscodon superciliosus Linnaeus, 1758 (Tropiduridae) for magicalreligious purposes in North and Northeastern of Brazil. Sitientibus série Ciências Biológicas 8: 257-258.

Alves, R.R.N. 2009. Fauna used in popular medicine in Northeast Brazil. Journal of Ethnobiology and Ethnomedicine 5: 1.

Alves, R.R.N. \& Alves, H. 2011. The faunal drugstore: animalbased remedies used in traditional medicines in Latin America. Journal of Ethnobiology and Ethnomedicine 7: 9.

Alves, R.R.N. \& Rosa, I.L. 2005. Why study the use of animal products in traditional medicines? Journal of Ethnobiology and Ethnomedicine 1: 5.

Alves, R.R.N. \& Rosa, I.L. 2006. From cnidarians to mammals: the use of animals as remedies in fishing communities in NE Brazil. Journal of Ethnopharmacology 107: 259-276.

Alves, R.R.N. \& Rosa, I.L. 2007a. Zootherapeutic practices among fishing communities in North and Northeast Brazil: a comparison. Journal of Ethnopharmacology 111: 82-103.

Alves, R.R.N. \& Rosa, I.L. 2007b. Zootherapy goes to town: the use of animal-based remedies in urban areas of NE and N Brazil. Journal of Ethnopharmacology 113: 541-555.

Alves, R.R.N. \& Santana, G.G. 2008. Use and commercialization of Podocnemis expansa (Schweiger 1812) (Testudines: Podocnemididae) for medicinal purposes in two communities in North of Brazil. Journal of Ethnobiology and Ethnomedicine 4: 3.

Alves, R.R.N. \& Souto, W.M.S. 2010a. Etnozoologia: conceitos, considerações históricas e importância. In: R.R.N. Alves, W.M.S. Souto \& J.S. Mourão (eds), A Etnozoologia no Brasil: importância, status atual e perspectivas. NUPEEA, Recife, p. 19-40.

Alves, R.R.N. \& Souto, W.M.S. 2010b. Panorama atual, avanços e perspectivas futuras para Etnozoologia no Brasil. In: R.R.N.
Alves, W.M.S. Souto \& J.S. Mourão (eds), A Etnozoologia no Brasil: importância, status atual e perspectivas. NUPEEA, Recife, p. 41-56.

Alves, R.R.N. \& Souto, W.M.S. 2010c. Desafios e dificuldades associadas às pesquisas etnozoológicas no Brasil. In: R.R.N. Alves, W.M.S. Souto \& J.S. Mourão (eds), A Etnozoologia no Brasil: importância, status atual e perspectivas. NUPEEA, Recife, p. 57-66.

Alves, R.R.N.; Rosa, I.L. \& Santana, G.G. 2007. The role of animal-derived remedies as complementary medicine in Brazil. BioScience 57: 949-955.

Alves, R.R.N.; Vieira, W.L.S. \& Santana, G.G. 2008. Reptiles used in traditional folk medicine: conservation implications. Biodiversity and Conservation 17: 2037-2049.

Alves, R.R.N.; Barbosa, J.A.A.; Santos, S.L.D.X.; Souto, W.M.S. \& Barboza, R.R.D. 2009a. Animal-based remedies as complementary medicines in the semi-arid region of Northeastern Brazil. Evidence-Based Complementary and Alternative Medicine: nep134.

Alves, R.R.N.; Neto, N.A.L.; Brooks, S.E. \& Albuquerque, U.P. 2009b. Commercialization of animal-derived remedies as complementary medicine in the semi-arid region of Northeastern Brazil. Journal of Ethnopharmacology 124: 600-608.

Alves, R.R.N.; Barboza, R.R.D. \& Souto, W.M.S. 2009c. Endangered Felidae used in traditional medicine. In: A. Columbus \& L. Kuznetsov (eds), Endangered Species: new research. Nova Science Publishers, Hauppauge, p. 343-356.

Alves, R.R.N.; Silva, C.C., Barboza; R.R.D. \& Souto, W.M.S. 2009d. Zootherapy as an alternative therapeutic in South America. Journal of Alternative Medicine Research 1: 2147.

Alves, R.R.N.; Mendonça, L.E.T.; Confessor, M.V.A.; Vieira, W.L.S. \& Lopez, L.C.S. 2009e. Hunting strategies used in the semi-arid region of northeastern Brazil. Journal of Ethnobiology and Ethnomedicine 5: 12.

Alves, R.R.N.; Souto, W.M.S. \& Barboza, R.R.D. 2010a. Primates in traditional folk medicine: a world overview. Mammal Review 40: 155-180.

Alves, R.R.N.; Barboza, R.R.D. \& Souto, W.M.S. 2010b. A global overview of canids used in traditional medicines. Biodiversity and Conservation 19: 1513-1522.

Ashwell, D. \& Walston, N. 2008. An overview of the use and trade of plants and animals in traditional medicine systems in Cambodia. TRAFFIC Southeast Asia, Greater Mekong Programme, Ha Noi.

Baker, F.C. 1941. A study of ethnozoology of the prehistoric indians of Illinois. Transactions of the American Philosophical Society 32: 51-77.

Brautigam, A.J.; Howes, T.; Hutton, H. \& Hutton, J. 1994. Analysis of the market for tigers, bears and musk deer in the Russian far east. Traffic Bulletin 15: 23-30.

Bye Jr, R.A. 1986. Voucher specimens in Ethnobiological studies and publications. Journal of Ethnobiology 6: 1-8.

Calixto, J.B. 2005. Twenty-five years of research on medicinal plants in Latin America: a personal view. Journal of Ethnopharmacology 100: 131-134.

Costa-Neto, E.M. 1999. Healing with animals in Feira de Santana City, Bahia, Brazil. Journal of Ethnopharmacology 65: 225- 
230.

Costa-Neto, E.M. 2000. A etnoentomologia no Brasil: um panorama bibliográfico. Biokos 14: 31-45.

Costa-Neto, E.M. 2005a. Animal-based medicines: biological prospection and the sustainable use of zootherapeutic resources. Anais da Academia Brasileira de Ciências 77: 3343.

Costa-Neto, E.M. 2005b. Entomotherapy, or the medicinal use of insects. Journal of Ethnobiology 25: 93-114.

Costa-Neto, E.M. \& Alves, R.R.N. 2010a. Estado da arte da zooterapia popular no Brasil. In: E.M. Costa-Neto \& R.R.N. Alves (eds), Zooterapia: os animais na medicina popular brasileira. NUPEEA, Recife, p. 13-54.

Costa-Neto, E.M. \& Alves, R.R.N. 2010b. Zooterapia: os animais na medicina popular brasileira. NUPEEA, Recife.

De Smet, P.A.G.M. 1991. Is there any danger in using traditional remedies? Journal of Ethnopharmacology 32: 43-50.

Debbie, N.G. \& Burgess, E.A. 2005. Against the Grain: trade in musk deer products in Singapore and Malaysia. WWF/ TRAFFIC Southeast Asia.

Dollo, M.; Gopi, G.V.; Teegalapalli, K. \& Mazumdar, K. 2010. Conservation of the orange-bellied Himalayan squirrel Dremomys lokriah using a traditional knowledge system: a case study from Arunachal Pradesh, India. Oryx 44: 573576.

El-Hani, C.N. \& Bandeira, F.P.S.F. 2008. Valuing indigenous knowledge: to call it "science" will not help. Cultural Studies of Science Education 3: 751-779.

Ellis, R. 2005. Tiger Bone \& Rhino Horn: the destruction of wildlife for traditional Chinese medicine. Shearwater Books/ Island Press, Washington, D.C.

Fabricant, D.S. \& Farnsworth, N.R. 2001. The value of plants used in traditional medicine for drug discovery. Environmental Health Perspectives 109: 69-75.

Farnsworth, N.R. 1988. Screening plants for new medicines. In: E.O. Wilson \& F.M. Peter (eds), Biodiversity. National Academy Press, Washington, D.C., p. 83-97.

Ferreira, F.S.; Brito, S.; Ribeiro, S.; Almeida, W. \& Alves, R.R.N. 2009a. Zootherapeutics utilized by residents of the community Poço Dantas, Crato - CE, Brazil. Journal of Ethnobiology and Ethnomedicine 5: 21.

Ferreira, F.S.; Brito, S.; Ribeiro, S.; Saraiva, A.; Almeida, W. \& Alves, R.R.N. 2009b. Animal-based folk remedies sold in public markets in Crato and Juazeiro do Norte, Ceara, Brazil. BMC Complementary and Alternative Medicine 9: 117.

Ferreira, F.S.; Brito, S.V.; Saraiva, R.A.; Araruna, M.K.A.; Menezes, I.R.A.; Costa, J.G.M.; Coutinho, H.D.M.; Almeida, W.O. \& Alves, R.R.N. 2010. Topical antiinflammatory activity of body fat from the lizard Tupinambis merianae. Journal of Ethnopharmacology 130: 514-520.

Ferreira, F.S.; Silva, N.L.G.; Matias, E.F.F.; Brito, S.V.; Oliveira, F.G.; Costa, J.G.M.; Coutinho, H.D.M.; Almeida, W.O. \& Alves, R.R.N. 2011. Potentiation of aminoglycoside antibiotic activity using the body fat from the snake Boa constrictor. Revista Brasileira de Farmacognosia 21(3): 503-509.

Fitch, H.S.; Henderson, R.W. \& Hillis, D.M. 1982. Exploitation of iguanas in Central America. In: G.M. Burghardt \& A.S. Rand (eds), Iguanas of the World: their behavior, ecology and conservation. Noyes Publications, New Jersey, p. 397-
415

Fragoso, J.M.V.; Bodmer, R.E. \& Silvius, K. 2004. Introduction - wildlife conservation and management in South and Central America: multiple pressures and innovative solutions. In: K. Silvius, R.E. Bodmer \& J.M.V. Fragoso (eds), People in Nature: wildlife conservation in South and Central America. Columbia University Press, New York, p. 1-8.

Holmstedt, B. \& Bruhn, J.G. 1983. Ethnopharmacology - a challenge. Journal of Ethnopharmacology 8: 251-256.

Hunn, E. 1992. The use of sound recordings as voucher specimens and stimulus materials in ethnozoological research. Journal of Ethnobiology 12: 187-198.

Hunter Jr, M.L. \& Gibbs, J.P. 2007. Species diversity. In: M.L. Hunter Jr. \& J.P. Gibbs (eds), Fundamentals of Conservation Biology. Wiley-Blackwell, Maden, p. 34-65.

Ingold, T. 1994. From trust to domination: an alternative history of human-animal relations. In: A. Manning \& J. Serpell (eds), Animals and Human Society: changing perspectives. Routledge, London, p. 1-22.

IBAMA (Instituto Brasileiro do Meio Ambiente e dos Recursos Naturais Renováveis) 2003. Lista das Espécies da Fauna Brasileira Ameaçadas de Extinção. Disponível em http:// www.ibama.gov.br/; acesso em 15 maio 2011.

IUCN (International Union for Conservation of Nature) 2009. Extinction Crisis Continues Apace. Disponível em http:// www.iucn.org/about/work/programmes/species/red_list/ ?4143/Extinction-crisis-continues-apace; acesso em 15 maio 2011.

IUCN (International Union for Conservation of Nature) 2010. IUCN Red List of Threatened Species. Disponível em http:// www.iucnredlist.org; acesso em 15 maio 2011.

Kahn, L.H. 2006. Confronting zoonoses, linking human and veterinary medicine. Emerging Infectious Diseases 12: 556561.

Kunin, W.E. \& Lawton, J.H. 1996. Does biodiversity matter ? Evaluating the case for conserving species. In: K.J. Gaston (ed.), Biodiversity: a biology of numbers and differences. Blackwell Science, Oxford, p. 283-308.

Lee, S.; Hoover, C.; Gaski, A. \& Mills, J. 1998. A World apart? Attitudes toward traditional Chinese medicine and endangered species in Hong Kong and the United States. TRAFFIC East Asia, TRAFFIC North America, and World Wildlife Fund, Washington, D.C.

Lev, E. 2000. The medicinal uses of animals and their products in the medieval Levant. Harefua 139: 483-487.

Lev, E. 2003. Healing with animals (zootherapy) from practical medieval medicine to present-day traditional medicine in the Levant. Journal of Ethnopharmacology 85: 107-118.

Liu, Q.; Yang, L. \& Tian, X. 2007. Podophyllotoxin: current perspectives. Current Bioactive Compounds 3: 37-66.

Loveridge, A.J.; Wang, S.W.;. Frank L.G. \& Seidensticker, J. 2010. People and wild felids: conservation of cats and management of conflicts. In: D.W. Macdonald \& A.J. Loveridge (eds), The Biology and Conservation of Wild Felids. Oxford University Press, Oxford, p. 161-197.

Macdonald, D.W.; Loveridge, A.J. \& Nowell, K. 2010. Dramatis personae: an introduction to the wild felids. In: D.W. Macdonald \& A.J. Loveridge (eds), The Biology and Conservation of Wild Felids. Oxford University Press, Oxford, p. 3-59.

Mahawar, M.M. \& Jaroli, D.P. 2006. Animals and their products 
utilized as medicines by the inhabitants surrounding the Ranthambhore National Park, India. Journal of Ethnobiology and Ethnomedicine 2: 46.

Mahawar, M.M. \& Jaroli, D.P. 2007. Traditional knowledge on zootherapeutic uses by the Sahari tribe of Rajasthan, India. Journal of Ethnobiology and Ethnomedicine 3: 6.

Mahawar, M.M. \& Jaroli, D.P. 2008. Traditional zootherapeutic studies in India: a review. Journal of Ethnobiology and Ethnomedicine 4: 17.

Marques, J.G.W. 1994. A fauna medicinal dos índios Kuna de San Blas (Panamá) e a hipótese da universalidade zooterápica. In: Anais da $46^{\circ}$ Reunião Anual da Sociedade Brasileira para o Progresso da Ciência, Vitória, Espírito Santo, Brasil, p. 324.

Marques, J.G.W. 1997. Fauna medicinal: recurso do ambiente ou ameaça à biodiversidade? Mutum 1: 4.

Mead, G.R. 1970. On the improper usage of common names when giving botanical data. American Antiquity 35: 108-109.

Miller, T.G. 1993. Environmental Science: sustaining the Earth. Wadsworth Publishing Company, Belmont.

Mills, J.A. \& Jackson, P. 1994. Killed for a Cure: a review of the worldwide trade in tiger bone. Traffic International, Cambridge.

Mittermeier, R.A.; Valladares-Pádua, C.; Rylands, A.B.; Eudey, A.A.; Butynski, T.M.; Ganzhorn, J.U.; Kormos, R.; Aguiar, J.M. \& Walker, S. 2007. Primates in peril: the world's 25 most endangered primates, 2004-2006. Primate Conservation 22: 1-40.

Moquin-Tandon, A. 1861. Elements of Medical Zoology. Bailliere, London.

Moura, F.B.P. \& Marques, J.G.W. 2008. Zooterapia popular na Chapada Diamantina: uma medicina incidental? Ciência \& Saúde Coletiva 13: 2179-2188.

Nguyen, M.L.T. 2005. Cultivated plant collections from market places. Ethnobotany Research \& Applications 33: 5-15.

Nijman, V. 2010. An overview of international wildlife trade from Southeast Asia. Biodiversity and Conservation 19: 11011114.

Oliveira, E.S.; Torres, D.F.; Brooks, S.E. \& Alves, R.R.N. 2010. The medicinal animal markets in the metropolitan region of Natal City, Northeastern Brazil. Journal of Ethnopharmacology 130: 54-60.

Patwardhan, B. 2009. Drug Discovery and Development: traditional medicine and ethnopharmacology perspectives. Disponível em http://www.scitopics.com/Drug_Discovery_ and_Development_Traditional_Medicine_and_ Ethnopharmacology_Perspectives.html; acesso em 21 ago. 2010.

Pieroni, A.; Giusti, M.E. \& Grazzini, A. 2002. Animal remedies in the folk medicinal practices of the Lucca and Pistoia Provinces, Central Italy. In: J. Fleurentin, J.M. Pelt \& G. Mazars (eds), Des Sources du Savoir aux Médicaments du Futur/From the Sources of Knowledge to the Medicines of the Future. IRD Editions, Paris, p. 371-375.

Pratt, M.L. 1992. Imperial Eyes: travel writing and transculturation. Routledge, London.

Quave, C.L.; Lohani, U.; Verde, A.; Fajardo, J.; Rivera, D.; Obón, C.; Valdes, A. \& Pieroni, A. 2010. A comparative assessment of zootherapeutic remedies from selected areas in Albania, Italy, Spain and Nepal. Journal of Ethnobiology 30: $92-125$.
Reed, H. 1995. Saiga antelope threatened by massive trade. TRAFFIC USA 14: 1-2.

Richmond-Coggan, L. 2006. Assessing Wildlife Distribution and Population Trends in the Greater Mara Ecosystem, Kenya: the synergistic effects of landscapes and threats. M.Sc. Thesis. University of Kent.

Rochfort, S.; Parker, A.J. \& Dunshea, F.R. 2008. Plant bioactives for ruminant health and productivity. Phytochemistry 69: 299-322.

Schipper, J.; Chanson, J.S.; Chiozza, F.; Cox, N.A.; Hoffmann, M.; Katariya, V.; Lamoreux, J.; Rodrigues, A.S.L.; Stuart, S.N.; Temple, H.J. \& al. 2008. The status of the world's land and marine mammals: diversity, threat, and knowledge. Science 322: 225-230.

Schnurrenberger, P.R. \& Hubbert, W.T. 1981. An Outline of the Zoonoses. Iowa State University Press, Ames.

Shepherd, C.R. 2001. Observations on wildlife trade at Golden Rock, Myanmar. TRAFFIC Bulletin 19: 7-10.

Shetty, P. 2010. Integrating Modern and Traditional Medicine: facts and figures. Disponível em http://www.scidev.net/en/ features/integrating-modern-and-traditional-medicine-factsand-figures.html; acesso em 21 ago. 2010.

Sodeinde, O.A. \& Soewu, D.A. 1999. Pilot study of the traditional medicine trade in Nigeria. TRAFFIC Bulletin 18: $35-40$.

Soejarto, D.D. 1996. Biodiversity prospecting and benefitsharing: perspectives from the field. Journal of Ethnopharmacology 51: 1-15.

Sousa, G.S. 1851.Tratado Descriptivo do Brazil em 1587. Tipographia Universal de Laemmert, Rio de Janeiro.

Souto, W.M.S.; Barboza, R.R.D.; Mourão, J.S. \& Alves, R.R.N. 2009. Zootherapy in Brazil: an urgent necessity of interdisciplinary studies. West Indian Medical Journal 58: 494-495.

Souto, W.M.S.; Mourão, J.S.; Barboza, R.R.D. \& Alves, R.R.N. 2011. Parallels between zootherapeutic practices in ethnoveterinary and human complementary medicine in NE Brazil. Journal of Ethnopharmacology 134: 753-767.

Stephenson, J. 1832. Medical Zoology, and Mineralogy; or Illustrations and Descriptions of the Animals and Minerals Employed in Medicine, and of the Preparations Derived from Them: including also an account of animal and mineral poisons. John Wilson, London.

Still, J. 2003. Use of animal products in traditional Chinese medicine: environmental impact and health hazards. Complementary Therapies in Medicine 11: 118-122.

Tabuti, J.R.S.; Dhillion, S.S. \& Lye, K.A. 2003. Ethnoveterinary medicines for cattle (Bos indicus) in Bulamogi county, Uganda: plant species and mode of use. Journal of Ethnopharmacology 88: 279-286.

TRAFFIC 2010. Biodiversity Indicators Partnership: biodiversity for food and medicine. Disponível em www.traffic.org/ medicinal-reports/traffic_pub_medicinal12.pdf; acesso em 15 maio 2011.

Van den Bos; R. \& de Jonge, F.H. 2005. Introduction. In: F.H. de Jonge \& R. van den Bos (eds), The Human-Animal Relationship: forever and a day. Royal Van Gorcum, Assen, p. 1-4.

Van, N.D.N. \& Tap, N. 2008. An Overview of the Use of Plants and Animals in Traditional Medicine Systems in Viet Nam. TRAFFIC Southeast Asia, Greater Mekong Programme, Ha 
Noi.

Vázquez, P.E.; Méndez, R.M.; Guiascón, Ó.G.R. \& Piñera, E.J.N. 2006. Uso medicinal de la fauna silvestre en los Altos de Chiapas, México. Interciencia 31: 491-499.

Vié, J.-C.; Hilton-Taylor, C. \& Stuart, S.N. 2009. Wildlife in a Changing World - an analysis of the 2008 IUCN Red List of Threatened Species. Lynx Edicions, Barcelona.

Wade, G. 2008. Gorilla Species Critically Endangered due to Loss of Habitats. Disponível em http://www.mediaglobal.org/ article/2008-10-19/gorilla-species-critically-endangered-dueto-loss-of-habitats; acesso em 15 maio 2011.

Walston, N. 2005. An overview of the use of Cambodia's wild plants and animals in traditional medicine systems. TRAFFIC Southeast Asia, Indochina.

Wingard, J.R. \& Zahler, P. 2006. Silent Steppe: the illegal wildlife trade crisis in Mongolia. World Bank, Washington, D.C.

World Health Organization 2002. WHO Traditional Medicine Strategy 2002-2005. WHO, Geneva. 